\title{
5 Medizin zwischen Arzt und Algorithmus - Digitale Revolution auch in der Versorgung?
}

\author{
Thomas Ballast
}

Die Zukunft ist digital. Daran zweifelt heute niemand. Traditionelle Märkte verändern sich massiv und durchleben einen grundlegenden Strukturwandel. Die Beispiele sind zahlreich: Das klassische Kaufhaus steht im Wettbewerb mit dem InternetVersandhandel. Reisen und Hotelbuchungen werden oft über das Internet abgewickelt statt im Reisebüro. Mit moderner Mobilität assoziieren viele Menschen heute nicht mehr die eine oder andere Automarke, sondern sogenannte Car-Sharing-Dienste. Nun sind Veränderungen in Gesellschaft und Wirtschaft kein neues Phänomen. Die Geschichte der Menschheit ist die Geschichte von Veränderungen. Ein revolutionäres Kennzeichen, das den derzeitigen Wandel ausmacht, ist jedoch die Geschwindigkeit, mit der diese Veränderungen von statten gehen. Die Digitalisierung wirkt wie ein Katalysator für den technischen Fortschritt.

Natürlich macht die Digitalisierung vor der Gesundheitsversorgung nicht halt. Vieles von dem, was heute schon in der Medizin möglich ist, war für die Menschen in der Mitte des 20. Jahrhunderts noch reine Science-Fiction. Bildgebende Diagnostik in 3D. Robotergestützte Operationen. Telemedizinisches Monitoring. Gentherapie. All das kannten die Menschen vielleicht aus Comics oder Serien wie „Star Trek“. Mit der (Lebens-)Wirklichkeit hatte das jedoch nichts zu tun. Wenn wir einen Blick in Zukunft wagen, dann können wir nur erahnen, was in zehn, 20 oder 30 Jahren medizinisch möglich sein wird. Fest steht jedoch: Das Tempo des medizinischen Fortschritts bestimmen ganz wesentlich die Entwicklungen in der Computerindustrie. IT-Lösungen nehmen einen immer größeren Stellenwert ein, wenn es um die medizinische Versorgung von Patienten geht. Daher ist es nicht verwunderlich, dass auch im Gesundheitswesen ein immenser Innovationsdruck von großen Computer- und Internetkonzernen ausgeht. 


\section{Das Gesundheitswesen für digitale Innovationen öffnen}

Neben den großen IT-Firmen wittern auch viele kleine Start-ups ihre Chance im Gesundheitsmarkt - mit zum Teil vielen interessanten Ideen. Längst ist so etwas wie Goldgräberstimmung entstanden. Und es sind vor allem die mobilen Endgeräte wie Smartphone oder Tablet, die den Start-ups den Zugang zur Gesundheit und der Digitalisierung insgesamt einen Schub gegeben haben. Sie haben nicht nur den Zugang zu Informationen grundlegend verändert, sondern auch den Umgang mit unseren persönlichen Daten. Das Problem im Gesundheitswesen ist nur, dass es heute noch keinen geregelten Rahmen gibt, wie gute digitale Versorgungsideen zügig den Weg ins System finden. Oftmals prallen hier zwei Welten aufeinander: Start-ups mit ihren kurzen Innovationszyklen auf der einen Seite und das korporatistisch verfasste Gesundheitswesen mit seinen zum Teil langwierigen Entscheidungsprozessen auf der anderen. Die Strukturen unseres Gesundheitssystems sind nicht auf die Geschwindigkeit der Digitalisierung vorbereitet. Die Folge ist, dass die Anbieter digitaler Versorgungsprodukte häufig auf den Zweiten Gesundheitsmarkt - den Markt der Selbstzahler - ausweichen.

Wenn aber die bestehenden Regelungen keinen adäquaten Zugang zum Ersten Gesundheitsmarkt bieten, ist es an der Zeit, über komplett neue Zulassungswege nachzudenken. Das ICES-Institut hat im Auftrag der Techniker Krankenkasse (TK) ein Verfahren vorgeschlagen, das die Vermeidung potenzieller Gesundheitsrisiken digitaler Versorgungsprodukte als wesentliches Kriterium für ein erstes, befristetes Zulassungsverfahren vorsieht. Das Risiko für die Nutzer/Patienten steigt mit dem Grad der Individualisierung von Informationen und mit dem Übergang von der Darstellung von Daten zur Ableitung von Therapieempfehlungen. Das vorgeschlagene Modell sieht deshalb vor, die Anforderungen im Zulassungsprozess an den Grad des möglichen Gesundheitsrisikos anzupassen.

\section{Der Digitalisierung sei Dank - Der Patient wird zum Partner auf Augenhöhe}

Es gilt der Spruch: Wenn der Wind der Veränderung weht, bauen die einen Mauern und die anderen Windmühlen. Leider gibt es im Gesundheitswesen noch zu viele, die die Digitalisierung mit Mauern aussperren möchten. DieTK gehört definitiv nicht dazu. Wir möchten die Tore des Gesundheitswesens für die Digitalisierung öffnen. Denn wir sind von den positiven Effekten für die Gesundheit überzeugt. Es wäre fahrlässig, auf die Chancen und Einsatzmöglichkeiten von E-Health, M-Health, Telemedizin und Co. für Prävention, Diagnostik, Therapie und auch Pflege zu verzichten. Ziel der TK ist es daher, die Medizin der Zukunft schon heute erlebbar zu machen.

Aus Sicht der TK hat die Digitalisierung das Potenzial, die medizinische Versorgung nicht nur effizienter zu machen, sondern auch die Qualität zu verbessern. Vor allem trägt sie dazu bei, die Kommunikation zu verändern - insbesondere die zwischen Arzt und Patient. Die Versicherten bzw. Patienten haben heute mithilfe digitaler Technik immer mehr Möglichkeiten, sich zu medizinischen Fragen zu informieren. Die Menschen sind nicht mehr nur passiv auf den Arzt und dessen Wissen angewiesen. Und die Versicherten nutzen diese Möglichkeiten. Sie wollen mehr Selbstverantwortung für sich und ihre Gesundheit übernehmen. Das führt dazu, dass immer mehr von 
ihnen entsprechend selbstbewusst gegenüber Ärzten und anderen Akteuren im Gesundheitswesen auftreten. Die Patienten verlassen sich nicht mehr allein auf das, was ihnen ihre Ärzte sagen. Die Folge ist: Wir erleben eine „(Halb-)Götterdämmerung in weiß“, die in Zukunft weiter zunehmen wird.

Immer mehr Menschen stellen außerdem infrage, ob wirklich jede Diagnose einen persönlichen Arztbesuch erfordert. Apps, E-Mails, soziale Netzwerke oder Video-Telefonie bieten praktische Alternativen, um mit Medizinern zu kommunizieren und ihren ärztlichen Rat einzuholen. Der physische (Arzt-)Kontakt wird dadurch entbehrlicher. All das rüttelt an bestehenden Restriktionen wie zum Beispiel das Fernbehandlungsverbot, das in der heute geltenden Form kaum Bestand haben wird.

\section{Daten sind nichts, Vernetzung ist alles}

Mithilfe der Sensorik und den Entwicklungen im Bereich der Bioelektronik lassen sich technisch immer mehr Daten erheben. Diese Daten werden gespeichert, weitergeleitet und ausgewertet. Cetreu dem Motto: Daten teilen, Informationen gewinnen. Dank digitaler Technik können Informationen zum Gesundheitszustand der Patienten und ihrer Krankheitshistorie allen, die darüber Bescheid wissen sollten, einfach und schnell sektorenübergreifend zur Verfügung gestellt werden. Krankheitsverläufe werden transparenter. Doppelte Dokumentationen können entfallen und Ärzte gewinnen mehr Zeit, sich um ihre Patienten zu kümmern. Allerdings brauchen diese Gesundheitsdaten einen sicheren Hafen.

Aus Sicht der TK sollte dieser Hafen eine elektronische Gesundheitsakte (eGA) sein, die niemand anderem als dem Versicherten gehört. Er allein muss das Recht haben, darüber zu entscheiden, welche Daten Eingang in die Akte finden, und wem er diese freigibt. Denn trotz des allgemeinen Interesses an mehr Transparenz in der medizinischen Versorgung darf man nicht vergessen, dass es allein seine Daten sind. Die TK arbeitet eigenständig an einer solchen Lösung für ihre Versicherten. Denn wir möchten nicht erst darauf warten, bis die gematik im Gesundheitswesen, die mit dem Aufbau einer flächendeckenden Telematikinfrastruktur beauftragt ist, die erforderlichen Beschlüsse für eine Patientenakte gefasst hat. Damit jedoch keine Insellösung geschaffen wird, soll die eGA, die die TK ihren Kunden anbieten wird, so ausgestaltet sein, dass sie an andere technische Entwicklungen und Angebote anknüpfen kann. Interoperabilität ist eine wichtige Voraussetzung für den Datentransfer im Gesundheitswesen.

Die Digitalisierung wird immer wieder mit dem Begriff „Disruption“ in Verbindung gebracht. Es geht um die Frage: Inwieweit beseitigt der digitale Wandel bestehende Strukturen und ersetzt sie durch neue? Vor allem besteht die Befürchtung, dass Computer den Menschen und seine Arbeit ersetzen könnten. Und in der Tat schreiten die Entwicklungen in der Computertechnologie immer weiter voran, sodass Computer wahrscheinlich eines Tages in der Lage sein werden, zumindest Teile der Arbeit des Arztes obsolet werden zu lassen. Big Data, Machine Learning, künstliche Intelligenz sind nur einige Stichworte, die den Fortschritt in der Computerindustrie beschreiben. Die Rechner werden immer leistungsfähiger. Sie sind bereits heute in der Lage, massive Mengen loser, unstrukturierter (Gesundheits-)Daten zu ver-und bearbeiten und „klug“ miteinander zu vernetzen - also zu strukturieren. Darüber hinaus können Computer heute schon Daten zum Teil in Echtzeit analysieren und mit der vorhan- 
denen medizinischen Literatur abgleichen, um daraus neue Informationen und Erkenntnisse zu generieren. Daten wandeln sich somit in medizinisches Wissen. Aber damit nicht genug: Sogenannte kognitive Systeme lernen inzwischen sogar aus Erfahrungen.

\section{Ist der Computer der bessere Arzt?}

Im Grunde macht der Computer nichts anderes als der Arzt: Er verbindet die zur Verfügung stehenden Daten und Informationen mit dem vorhandenen Wissen, um eine optimale Therapieentscheidung zu treffen. Allerdings können Computer sehr viel mehr Daten und Wissen in sehr viel kürzerer Zeit bearbeiten, als dies ein Arzt kann. Was dem Computer jedoch zweifelsohne fehlt, ist Empathie. Deshalb können und möchten sich viele noch nicht vorstellen, dass der Computer zur entscheidenden Instanz der medizinischen Versorgung aufsteigt. Gleichwohl ist die Wahrscheinlichkeit, dass Computer auf lange Sicht auch empathisch agieren und reagieren können, relativ hoch. Spätestens dann wird sich zeigen, ob die Patienten bei Fragen zu Diagnostik und Therapie eher einem Menschen oder einer Maschine vertrauen werden.

Die Digitalisierung und mit ihr die Fortschritte in der Computertechnologie werden die medizinische Versorgung nachhaltig verändern. Die große Chance für die medizinische Versorgung besteht darin, dank komplexer Daten- und Wissensanalysen die evidenzbasierte mit der personalisierten Medizin zu kombinieren und zu versöhnen. Wenn dies flächendeckend gelingt, ist das die wahre digitale Revolution in der Versorgung. Als moderner Gesundheitsdienstleister und Interessenvertreter ihrer Versicherten sieht die TK ihre Aufgabe darin, die Realisierung dieses Ziels mit Nachdruck zu unterstützen. 